Mini Review Article

\title{
The Duality of AIM2 Inflammasome: A Focus on its Role in Autoimmunity and Skin Diseases
}

\author{
Miriam Canavese \\ The Basil Hetzel Institute for Translational Health Research, Discipline of Surgery, University of Adelaide, Australia
}

\author{
Article history \\ Received: 08-03-2016 \\ Revised: $16-03-2016$ \\ Accepted: 18-03-2016 \\ Email: miriamcanavese@yahoo.com
}

\begin{abstract}
Understanding the inflammasome biology is one of the most exciting challenges in immuno-pharmacology. The role of the inflammasomes has been recognized in the host defense mechanism against invading pathogens and in the development of several conditions, such as cancer, auto-inflammatory, autoimmune, metabolic and neurodegenerative disorders. DNA recognition by the cells is a crucial immunological step leading to the initiation of an innate immune response. Absent in Melanoma 2 (AIM2) is a cytoplasmic sensor that perceives double-stranded DNA of microbial or host origin. Once the DNA is bound, AIM2 assembles a multiprotein complex named inflammasome, which drives pyroptosis and proteolytic cleavage of pro-IL-1 $\beta$ and pro-IL-18 pro-inflammatory cytokines, leading to a protective inflammasome-mediated host response. However, improper recognition of self-DNA by AIM2 triggers deleterious inflammatory responses, leading to systemic inflammation and several pathological conditions. Therefore, understanding the mechanisms of AIM2-inflammasome-mediated inflammation will provide an essential knowledge base to develop new successful therapeutic strategies to cure the outlined pathologies in which AIM2- inflammasome activation plays a key role, as well as to guide clinical practice. This mini-review provides an overview on the latest research findings on AIM2 inflammasome, with particular focus on its role in autoimmunity and skin disorders. An update on its therapeutic implications has also been documented.
\end{abstract}

Keywords: Absent in Melanoma 2 (AIM2), Inflammasome, Autoimmune Disorders, Psoriasis, Inflammation

\section{Introduction}

The inflammasomes are innate immune system sensors that control the activation of caspase-1 and promote inflammation towards pathogens and molecules derived from host proteins (Guo et al., 2015). They have been associated to a variety of auto-inflammatory and auto-immune diseases, as well as neurodegenerative and metabolic disorders (Strowig et al., 2012). In the initiation of the inflammatory process, inflammasomes play key roles in causing, contributing or amplifying the pathology in response to the host-derived factors (Guo et al., 2015).

After sensing certain stimuli the appropriate NODLike Receptor (NLR) or the non-NLR Absent in Melanoma 2 (AIM2) can oligomerize to be a caspase-1 activating scaffold. The active caspase- 1 consequently cleaves the pro-inflammatory IL-1 family of cytokines into their bio-active forms (IL-1 $\beta$ and IL-18), causing pyroptosis, defined as a type of inflammatory cell death (Lamkanfi and Dixit, 2012; Strowig et al., 2012). The activation of the inflammasome is therefore a pivotal process mediated by the innate immune system. Recent findings have improved the understanding of this mechanism (Guo et al., 2015).

Over the past 10 years, at least 22 NLRs in humans and 34 members in mice have been discovered and widely described (Ting et al., 2008). A functional subgroup of NLRs has been identified as driving the formation of the inflammasome (Guo et al., 2015). The majority of the inflammasomes characterized so far, show an NLR protein, such as NLRP1, NLRP2, NLRP3, NLRP6, NLRP7, NLRP12, or NLRC4 (NLR-and caspase-activating recruitment domain-containing 4) (de Rivero Vaccari et al., 2014). In addition to the NLR 
inflammasomes, the non-NLR-AIM2, has been recognized as a member of the AIM2-Like Receptors (ALRs), consisting of a pyrin and Hin domains, which are essential for inflammasome activation induced by double-stranded DNA (dsDNA) (Liu et al., 2015).

The assemblage of an inflammasome complex compels cytosolic sensing of pathogen-associated molecular patterns by a nucleotide-binding domain and leucine-rich repeat receptor (NLR) or Absent in Melanoma 2 (AIM2)-Like Receptors (ALR) (Man and Kanneganti, 2015). To catalyze the proteolytic cleavage of pro-interleukin (IL)-1 $\beta$ and pro-IL-18, which results into pyroptosis, NLRs and ALRs engage caspase-1, through the adapter protein apoptosis-associated specklike protein, containing a CARD (ASC), (Man and Kanneganti, 2015). To become activated AIM2 or NLRP3 require the polymerization of the $\mathrm{N}$ terminal pyridine domain (PYD) of ASC ( $\mathrm{Lu}$ et al., 2014; Cai et al., 2014), which, as final step, leads to the formation of a single and distinct inflammasome speck that could be detected in primary macrophages and dendritic cells (DCs) (Man et al., 2014; 2015a; Belhocine and Monack, 2012). Recently Bakele and colleagues showed that human neutrophils also express the key components of the NLRP3 and AIM2 inflammasome machinery in different intra-cellular compartments and they are capable to release key cytokines. This findings add valuable knowledge to the well known cytoplasmic localization, described for a variety of cell types (Schroder and Tschopp, 2010), indicating that neutrophils are able to regulate their inflammasomes between intracellular stores, as secretory vesicles and the surface localization. Whether the neutrophils release the vesicles, stored in the inflammasome compartments, into the extracellular microenvironments needs to be further elucidated (Bakele et al., 2014). Adamczak et al. (2014) reported the presence of a functional AIM2-inflammasome in cortical neurons, which senses aberrant dsDNA through a mechanism leading to neural pyropotosis.

However, inflammasome activation occurs when the scaffold protein senses or binds its activating stimuli. This mechanism is now being clarified for certain inflammasome proteins (Vanaja et al., 2015): prominent among these are the roles of NLRC4, NAIP, ASC and AIM2 (Guo et al., 2015). The identification of AIM2, as the sensor that triggers inflammasome activation, pyroptosis and release of key cytokines in response to intracellularly delivered dsDNA, has happened in 2009 (Hornung et al., 2009; Fernandes-Alnemri et al., 2009; Burckstummer et al., 2009; Roberts et al., 2009). AIM2 recognizes dsDNA in a sequence-independent manner. However, the DNA sequence should be at least 80 base pairs in length (Jin et al., 2013). The clarification of the crystal structure of AIM2 had an impact on the explanation of its activation mechanism (Man et al., 2016).
This mini-review summarizes the latest research findings on the AIM2 inflammasome, focusing on its role in autoimmunity and skin diseases and discussing its therapeutic implications.

\section{The Duality of AIM2: An Overview}

Inflammation is a protective immune-response started by the innate immune system in response to harmful stimuli, such as microbes, dead cells, irritants and it is firmly regulated by the host. A lack of inflammation can lead to infections, while excessive inflammation can cause chronic or systemic inflammatory diseases (Guo et al., 2015). Innate immune detection and subsequent immune response depends on the initial recognition of pathogen specific molecular motifs (Smith and Jefferies, 2014). Unknown nucleic acids are key molecules recognized by the immune system, recognition of which occurs mainly through specific receptors, including members of Toll-like receptors, AIM2-like receptors, RIG-I-like receptors and intracellular DNA receptors (Smith and Jefferies, 2014). However, a wide range of pathogens is sensed by AIM2 in mammalian cells.

As shown in Fig. 1, recognition of dsDNA from pathogens by AIM2 heads to protective inflammasomemediated host responses. In contrast, improper recognition of cytoplasmic self-DNA by AIM2 leads to the development of several pathologies, such as psoriasis, dermatitis, arthritis and other autoimmune and inflammatory diseases (Man et al., 2016).

In the first case, during infection of a host cell, microbial DNA and other pathogen-associated molecular patterns are released into the cytoplasm, where they are recognized by cytoplasmic DNA sensors, such as AIM2. AIM2 has been shown to provide immune-surveillance to several pathogenic bacteria like Listeria monocytogenes, Streptococcus pneumonia, Staphilococcus aureus and many others (Rathinam et al., 2010; Fernandes-Alnemri et al., 2010; Kim et al., 2010; Warren et al., 2010; Hanamsagar et al., 2014; Tsuchiya et al., 2010; Man et al., 2016). These pathogens activate AIM2 via a 'non canonical' pathway owing to its requirement for type I IFN, analogous to the non-canonical NLRP3 inflammasome pathway (Man et al., 2016) and they must escape the vacuole and undergo bacteriolysis in order to induce the activation of the AIM2 inflammasome (Fang et al., 2011; Kim et al., 2010; Tsuchiya et al., 2010). Some bacteria have evolved virulence determinants to prevent release of DNA to avoid cytoplasmic and clearance by inflammasomes (Crane et al., 2014; Dotson et al., 2013), however there is limited evidence to support the existence of mechanisms used by bacteria to evade or inhibit activation of AIM2, which is overall an extraordinary antimicrobial machinery (Man et al., 2016). 


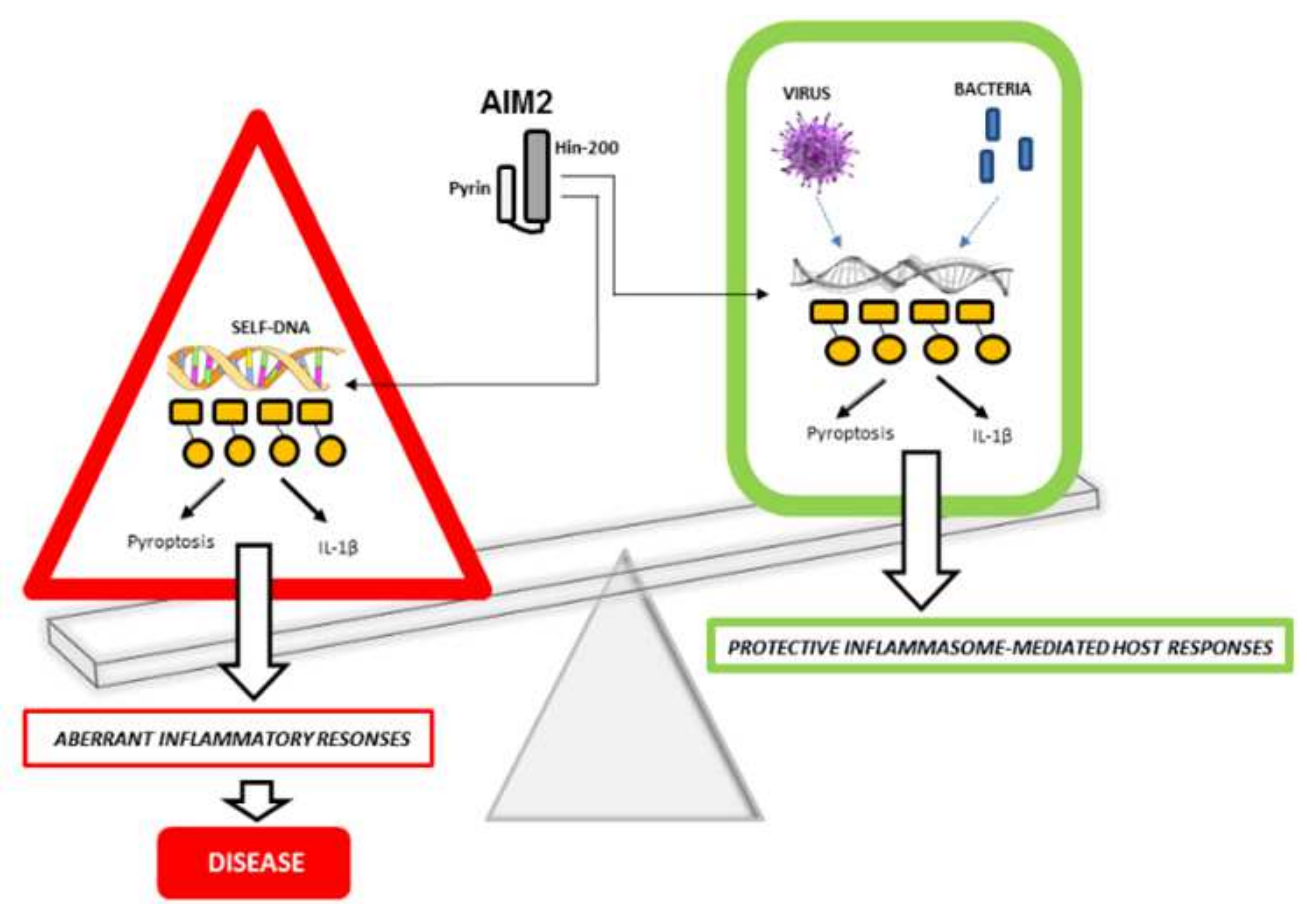

Figure 1. The yin and yang of AIM2 inflammasome. Simplified representation of AIM2 inflammasome activation induced by cytoplasmic dsDNA. AIM2 is formed by a N-terminal pyrin domain and a C-terminal HIN-200 domain, which together form an intramolecular complex that is maintained in an auto-inhibitory state. Upon binding to DNA, AIM2 assembles a multiprotein complex named inflammasome, which catalyzes proteolytic cleavage of pro-IL-1 family cytokines and drives pyropoptosis. Recognition of dsDNA from microbes by AIM2 leads to protective inflammasome-mediated host responses, whereas inappropriate recognition of self-DNA by AIM2 triggers aberrant inflammatory responses, leading to autoimmune diseases, psoriasis, dermatitis, chronic inflammatory diseases, neuroinflammation and metabolic disorders. Therefore, a deep understanding of the balance between the beneficial and the detrimental effects of the inflammasome activation is essential.

Inflammasome responses play also an important role in the host protection against viruses (Kanneganti, 2010; Lupfer et al., 2015). Murine cytomegalovirus infections (MCMV) lead to a 'canonical' activation of the AIM2 inflammasome, which doesn't require the type I IFN pathway (Rathinam et al., 2010). Recently, Zhen and colleagues showed a reduced expression of IL-1 $\beta$ and IL-18 and caspase-1 through siRNA-mediated silencing of the gene encoding AIM2 in the human glomerular mesangial cell line infected with hepatitis B virus (Zhen et al., 2014). Whether, AIM2 recognizes directly viral DNA derived from hepatitis B virus is still elusive: probably the viral DNA binds to AIM2 to trigger inflammasome activation, but the precise molecular mechanisms is not clear (Man et al., 2014).

Therefore, to date there is evidence in the literature to indicate that only MCMV, vaccinia viruses and human papilloma viruses induce inflammasome responses in a AIM2-dependent manner (Hornung et al., 2009; Rathinam et al., 2010; Reinholz et al., 2013) and it is known that AIM2 does not respond to all DNA viruses (Man et al., 2016). However, has been reported a role for AIM2 in driving IL-1 $\beta$ secretion in response to RNA viruses: silencing of genes encoding AIM2 and caspase- 1 reduces proteolytic cleavage and release of
IL-1 $\beta$ in human dermal fibroblasts infected with the RNA virus Chikungunya (Ekchariyawat et al., 2015). The mechanism by which AIM2 might sense RNA viruses is still unclear.

Additional to bacteria and viruses AIM2 mediates pathogen recognition of and host defense to the fungal pathogen Aspergillus fumigatus and the Plasmodium berghei ANKA (Karki et al., 2015; Kalantari et al., 2014). It has been shown, that mice lacking both AIM2 and NLRP3, ASC or caspase-1 and infected with $A$. fumigatus are more susceptible than infected WT (Karki et al., 2015). The requirement for dual sensing of pathogens by both AIM2 and NLRP3 has also been observed in mouse bone marrow-derived macrophages stimulated with Plasmodium berghei ANKA infected redblood cells (Kalantari et al., 2014). There is evidence that $A$. fumigatus genomic DNA, transfected into the cell cytoplasm as well as for the Plasmodium falciparum genomic DNA, transported into the cytoplasm by hemozoin are recognized by AIM2 directly (Kalantari et al., 2014; Karki et al., 2015). Further studies to elucidate the mechanisms involved are warranted.

AIM2 also contributes to the control of excessive inflammation in a complex, context-dependent way (Zitvogel et al., 2012). For example, it has been shown 
that AIM2 is implicated in inflammation and cell death of the brain. Cerebrospinal Fluid (CSF) of patients with traumatic brain injury often contains cell-free DNA fragments rather than the CSF from non-trauma patients and this correlates with mortality (Adamczak et al., 2014; Campello Yurgel et al., 2007). Adamczak and colleagues have demonstrated that human embryonal cortical neurons express functional AIM2 inflammasome, they release IL-1 $\beta$ and they undergo pyroptosis upon AIM2 activation with poly (dA:dT) transfection. Therefore, when exposed to the CSF of traumatic brain injury patients, they showed AIM2 upregulation and caspase-1 activation compared with embryonic cortical neurons that had been exposed to the CSF of non-trauma patients, indicating that CSF from injured patients is immunogenic and may cause pyropotosis in neighboring cells (Adamczak et al., 2014). These findings establish neuronal pyroptosis as a neuronal cell death mechanism, induced by activation of the AIM2 inflammasome (Adamczak et al., 2014). In the context of cancer, beyond its role in killing infected macrophages, pyroptosis may participate to the cellautonomous tumor suppression. This suggests that the inflammasomes can positively influence the cellautonomous death pathways and anticancer immunesurveillance, but they can also induce autocrine or paracrine processes that favor carcinogenic inflammation, tumor growth, metastasis and angiogenesis (Zitvogel et al., 2012; Miao et al., 2011). In support of the role of AIM2 inflammasome as a suppressor of cancer, it has been found that colorectal cancer patients, whose tissues showed a reduced AIM2 expression have a poorer prognosis compared to those with an up-regulation of AIM2 (Dihlmann et al., 2014). Moreover, AIM2 downregulation has also been reported in prostate cancer (Ponomareva et al., 2013), whereas increased expression has been detected in nasopharyngeal carcinoma (Wang et al., 2012; Chen et al., 2012), oral squamous cell carcinoma (Kondo et al., 2012) and lung adenocarcinoma (Kong et al., 2015), suggesting that the differential expression of AIM2 in a range of tumor tissues could relate to its possible unique role in different types of cancer (Man et al., 2016). The mechanism by which AIM2 regulates tumorigenesis has been demonstrated by Wilson et al. (2015) and described in a mouse model of colitis-associated colorectal cancer (Man et al., 2015b). The above-mentioned studies have demonstrated that AIM2 works independently of the inflammasome to prevent colorectal cancer. Although differential secretion of the key cytokines, including TNF $\alpha$ and IL-6, was not observed among WT and Aim2/mice, proliferation of enterocytes was more pronounced in Aim2\%-mice (Man et al., 2015b; Wilson et al., 2015). Wilson and colleagues discovered a DNA-PK, a kinase that can phosphorylate and activate AKT, as a binding partner of AIM2, through which, AIM2 suppresses
DNA-PK activation (Wilson et al., 2015; Feng et al., 2004; Lu et al., 2006). Very recently, Man and colleagues also showed that a reciprocal exchange of the microbiota between Aim $2 \%$ and WT mice could cause a decrease in tumorigenesis in Aim $2 \%$ and an increase in tumorigenesis in WT mice (Man et al., 2015b). Collectively these studies provide insights into the function of AIM2 as tumor suppressor, especially in colorectal cancer. However, as discussed above, AIM2 inflammasome dysregulation, in terms of incorrect recognition of cytoplasmic self-DNA is reported to result in auto-inflammatory and auto-immune diseases (Smith and Jefferies, 2014; Choubey and Panchanathan, 2008).

\section{AIM2 in Autoimmunity and Skin Diseases}

The presence of altered self-DNA may be sensed in the nuclear compartment of cells, leading to inflammation and auto-immune responses (Choubey and Panchanathan, 2008; Fish, 2008). Systemic autoimmune diseases include Sjogren's Syndrome (SS), Systemic Lupus Erythematosus (SLE), Rheumatoid Arthritis (RA) and Systemic Sclerosis (SSc) (Choubey and Panchanathan, 2008). These disorders are characterized by self-antigen-driven immune responses that target host tissues and organs for damage (Crispin et al., 2010). Patients with systemic auto-immune diseases exhibit the so called "IFN-signature", since they show elevated serum levels of pro-inflammatory cytokines, such as IL-1, TNF $\alpha$, IFNs (Apostolidis et al., 2011; van der Pouw Kraan et al., 2007). It has been demonstrated, that mature SLE neutrophils, which are primed by increased levels of type I IFN, die when they are exposed to SLE-derived anti-ribonucleoprotein antibodies. Death of neutrophils leads to the release of neutrophil extracellular traps, containing genomic DNA (Garcia-Romo et al., 2011; Lande et al., 2011). It has been recently shown by Bakele and colleagues that human neutrophils express the machinery of NOD-like receptor family and AIM2 inflammasome (Bakele et al., 2014). Therefore, upon sensing self-derived DNA, the DNA receptors begin the immune responses (Choubey and Panchanathan, 2008). Several studies also indicate that lupus-associated pathogenesis includes an impaired clearance of dead cellular debris followed by an aberrant activation of the immune system (Baccala et al., 2009; Munoz et al., 2010). As a consequence, it has been proposed that activation of the innate immune responses, which are initiated by DNA sensors, such as AIM2, in cells contributes to the inflammatory responses in Systemic Lupus Erythematosus (SLE) (Munoz et al., 2010). In SLE, self-DNA is often complexed with nuclear antigens and the corresponding auto-antibodies (Marshak-Rothstein, 2006). Thus, the recognition of selfDNA by immune cells has a critical role in a feed forward loop, which promotes auto-antibody production, triggers 
innate immune responses and associated immunopathology in SLE and possibly other autoimmune disorders (Choubey and Panchanathan, 2008; Munoz et al., 2010; Baccala et al., 2007). Interestingly, a recent study by Kahlenberg and colleagues has demonstrated that expression of AIM2 gene is induced by IFN- $\alpha$ in Endothelial Progenitor Cells (EPCs) and Circulating Angiogenic Cells (CACs) (Kahlenberg et al., 2011). Considering the increase of premature atherosclerosis, associated with endothelial dysfunction, up to 50-fold, in SLE patients (Kahlenberg et al., 2011), it has been proposed that an imbalance between endothelial cell damage and repair, triggered by the enhanced inflammasome activity, may lead to a faster atherosclerosis through increased IL-18 activation (Kahlenberg et al., 2011; Kaplan, 2011). In addition, several studies demonstrated that the expression of the gene encoding AIM2 is increased in immune cells of male patients with SLE and both increases and decreases in AIM2 expression have been observed in female patients (Yang et al., 2015; Kimkong et al., 2009). Further, DNA methylation of the gene encoding AIM2 is decreased in patients with SLE compared with their healthy siblings, suggesting that epigenetic changes could also contribute to the development of this disease (Javierre et al., 2010).

The inability to degrade self-DNA also play a role in the pathogenesis of autoimmune polyarthritis (Man et al., 2016). It has been shown by Kawane and colleagues that mice lacking the lysosomal endonuclease DNAse II (Dnase $I \Gamma^{-}$mice) are embryonically lethal, due to the impaired ability to degrade self-DNA by macrophages (Kawane et al., 2001). Genomic deletion of type I IFN Receptor (IFNAR) rescued Dnase $\mathrm{II}^{-/-}$mice from embryonic lethality (Yoshida et al., 2005), but the mice lacking both IFNAR and DNAse II (Dnase II Ifnar ${ }^{-/-}$ mice) would develop polyarthritis (Kawane et al., 2001). Interestingly, Baum and colleagues demonstrated a peculiar role for the AIM2 inflammasome in arthritis pathogenesis. The group showed that genomic deletion of AIM2 and STING halted inflammasome activation, macrophage infiltration in the joint and consequently the development of arthritis in Dnase $\mathrm{II}^{-/-} \mathrm{Ifnar}^{-/-}$mice (Baum et al., 2015; Jakobs et al., 2015). This suggests that multiple DNA sensors might contribute to the inappropriate DNA recognition leading to the pathogenesis of a clinical disease (Man et al., 2016).

Accumulated DNA could result into an endogenous danger signal and it has been shown to trigger AIM2dependent release of IL-1 $\beta$ from keratinocytes, contributing to the pathogenesis of psoriasis (Dombrowski et al., 2011). Dombrowski and colleagues demonstrated the presence of cytosolic DNA and subsequent up-regulation of AIM2 in keratinocytes of psoriatic lesions versus healthy controls. Furthermore, they showed that cathelicidin LL-37, upon internalization into the cytosol of keratinocytes, was able to neutralize the cytosolic DNA and its pro-inflammatory effect, interfering with AIM2 activation and therefore acting as a physiologic inhibitor of AIM2 inflammasome (Dombrowski et al., 2011; 2012). In addition, de Koning et al. (2012) observed a strong epidermal upregulation of AIM2 protein expression in psoriasis, atopic dermatitis (AD), venous ulcera, contact dermatitis and experimental wounds, highlighting the dynamics of epidermal AIM2 expression and showing an important induction of Langherans cells and melanocyte-restricted expression in sub-populations of epidermal keratinocytes under inflammatory conditions, compared to normal epidermis. The remarkable increase of AIM2 expression in keratinocytes at site of acute and chronic skin barrier disruption-related inflammation, indicates a role for AIM2 inflammasome in both antimicrobial defense and also sustained chronic inflammation (de Koning et al., 2012). However, Kopfnagel and colleagues obtained contrasting results, suggesting that there is no intrinsic abnormality concerning the expression of AIM2 in keratinocytes of AD or psoriatic patients versus healthy controls, pointing out a general role of AIM2 in skin defense (Kopfnagel et al., 2011). Further investigations would be necessary to elucidate the contribution of AIM2 in inflammatory skin conditions. However, AIM2 up-regulation upon skin barrier disruption serves as first line of defense against invading pathogens (Fernandes-Alnemri et al., 2009; 2010, Hornung et al., 2009, Kim et al., 2010, Rathinam et al., 2010). This could also favor wound healing, however, if the barrier impairment persists, AIM2-induced IL-1 $\beta$ activation could promote an inflammatory loop in chronic skin disorders, such as psoriasis, AD and even venous leg ulcers (de Koning et al., 2012).

\section{Therapeutic Implications of AIM2 Inflammasome}

Although the therapeutic inhibition of the inflammasomes has to be balanced against its beneficial contribution, it is well known that inflammasome dysregulation leads to the pathogenesis of several diseases, including neurodegenerative and metabolic disorders as well as auto-inflammatory and autoimmune diseases. Thus, the development of new targeted drugs against the inflammasomes would be necessary for the treatment of these pathologies. The majority of available small-molecules inhibitors target NLPR3 inflammasome, but not NLRC4 or AIM2. The therapies that modulate either the NLPR3 inflammasome complex itself and the two cytokines, which is responsible for activating, are broadly detailed and recently reviewed already by Ozaki et al. (2015) and Baldwin et al. (2015). Here, a focus and an update on the AIM2 inflammasome-targeted molecules is documented and summarized in Table 1. 
Table 1. Therapeutic agents targeting AIM2- inflammasome components and their clinical status

\begin{tabular}{|c|c|c|}
\hline Therapeutic agent & Target & Clinical Status/Use \\
\hline Parthenolide & $\begin{array}{l}\text { AIM2 (indirectly) } \\
\text { Caspase-1 } \\
\text { ASC pyropoptosome formation }\end{array}$ & $\begin{array}{l}\text { Not suitable for clinical trials due to } \\
\text { its poor bioavailability and solubility }\end{array}$ \\
\hline Bay 11-7082 & $\begin{array}{l}\text { AIM2 (indirectly) } \\
\text { ASC pyropoptosome formation }\end{array}$ & $\begin{array}{l}\text { Approved } \\
\text { Systemic lupus erythematosus }\end{array}$ \\
\hline Cholesterol 25-hydroxylase (Ch25h) & $\begin{array}{l}\text { AIM2 (indirectly) } \\
\text { IL-1 } \beta \text { (regulate Il1b transcription) }\end{array}$ & N/A \\
\hline PRT062607 (Biogen, Idec, & AIM2 (indirectly) & \\
\hline Cambridge MA, USA) & $\begin{array}{l}\text { Tyrosine kinases } \\
\text { ASC speck formation }\end{array}$ & Under evaluation \\
\hline R-788 (AstraZeneca) & $\begin{array}{l}\text { AIM2 (indirectly) } \\
\text { Tyrosine kinases }\end{array}$ & $\begin{array}{l}\text { Abandoned } \\
\text { for low specificity and high toxicity }\end{array}$ \\
\hline Pralnacasan (VX-740) & $\begin{array}{l}\text { AIM2 (indirectly) } \\
\text { Caspase-1 }\end{array}$ & $\begin{array}{l}\text { Tested in clinical trials for RA, but outcome } \\
\text { of the clinical trial is not reported }\end{array}$ \\
\hline Cys-LT-receptor (Bayer AG, Leverkusen, & AIM2 (indirectly) & Approved \\
\hline Germany/US Patent 7,498,460, 2009) & ASC oligomerization & Allergic rhinitis, asthma, nasal polyposis \\
\hline ASC monoclonal Ab (clone $23-4$ & AIM2 (indirectly) & $\mathrm{N} / \mathrm{A}$ \\
\hline MBL, Nagoya, Japan) & ASC inhibition & Experimental research use \\
\hline Synthetic Oligodeoxynucleotides (ODN) & AIM2 & N/A \\
\hline containing suppressive TTAGGG motifs & $\begin{array}{l}\text { ASC dimerization } \\
\text { AIM2 inflammasome assembly }\end{array}$ & Experimental research use \\
\hline Bromoxone & $\begin{array}{l}\text { AIM2 (indirectly) } \\
\text { Caspase-1 } \\
\text { ASC }\end{array}$ & $\mathrm{N} / \mathrm{A}$ \\
\hline Probenecid & $\begin{array}{l}\text { AIM2 (indirectly) } \\
\text { Neural pyroptosis }\end{array}$ & $\begin{array}{l}\text { Approved } \\
\text { (under investigation its use to inhibit neural } \\
\text { pyroptosis during AIM2 inflammasome stimulation) }\end{array}$ \\
\hline
\end{tabular}

Abbreviations: AIM2, Absent in Melanoma 2; ASC, apoptosis-related speck-like protein containing a caspase recruitment domain; Cys-LTreceptor, cystenyl leukotriene, RA, rheumatoid arthritis; IL-1, Interleukin-1; The table summarizes the most recent therapeutic agents targeting the AIM2-inflammasome components described in the literature, therefore it is not exhaustive. Each therapeutic agent listed in the table has been detailed and referenced in the text.

Several small-molecule inhibitors targeting NLRP3, NLRP1, NLRC4 and AIM2 have been characterized and widely described in (Ozaki et al., 2015), even if their potency for in vivo usage needs further evaluation. The large majority of these are pharmacologic inhibitors that have been repurposed to target the inflammasome (Guo et al., 2015) and they include: Parthenolide (Juliana et al., 2010), Bay 11-708 (Juliana et al., 2010), CRID3 (Coll et al., 2011), Auranofin (Isakov et al., 2014), Isoliquiritigenin (Honda et al., 2014), 3,4methylenedioxy- $\beta$-nitrostyrene ( $\mathrm{He}$ et al., 2014), Cyclopentenone prostaglandin $15 \mathrm{~d}-\mathrm{PJ}_{2}$ (Maier et al., 2015) and 25-Hydroxycholesterol (25-HC) (Reboldi et al., 2014). Moreover, type I interferon has been shown to also suppress inflammasome activation with a poorly understood mechanism (Guarda et al., 2011). However, recently it has been demonstrated that an IFN-stimulated gene product, cholesterol 25-hydroxylase (Ch25h), antagonizes both Illb transcription and NLRP3, NLRC4 and AIM2 inflammasome activation, indicating that $\mathrm{Ch} 25 \mathrm{~h}$ has a broad inhibitory activity of multiple inflammasomes (Reboldi et al., 2014).
Interestingly, Hara et al. (2013) observed that, upon NLRP3 and AIM2 inflammasome activation, the tyrosine kinases Syk and Jnk regulate ASC speck formation. Phosphorylation of ASC is critical for inflammasomes aggregation, consequent pro-caspase-1 recruitment and cytokines release (Hara et al., 2013). These findings supported a growing interest in Syk and Jnk in the inflammatory response. Accordingly, several drugs, targeting these kinases have been recently tested in patients suffering from severe auto-inflammation (Grimminger et al., 2010; Laudisi et al., 2014). Meanwhile a promising candidate (PRT062607, Biogen Idec, Cambridge, MA, USA) is under evaluation, previous Syk inhibitors, such as AstraZeneca, R-788, showed low specificity and high toxicity, which forced to abandon their development (Genovese et al., 2011; Flight, 2012). Regardless, Hara and colleagues have showed that specific inhibition of phosphorylated ASC could represent an alternative means to enable suppression of caspase-1 activation and the associated pathological consequences (Laudisi et al., 2014). To this end, Pralnacasan (VX-740, Vertex Pharmaceuticals, Inc. 
Cambridge, MA, USA) is a specific caspase-1 inhibitor, which showed promising in animal model of osteoarthritis, but, unfortunately its toxicity was too high for clinical development (Rudolphi et al., 2003). Overall, this approach may enable more selective blocking of IL1 maturation and release, without the toxicity associated with kinase inhibition (Laudisi et al., 2014). Interestingly, a cysteinyl leukotriene receptor antagonist developed by Bayer Pharmaceuticals (Bayer AG, Leverkusen, Germany/Härter et al, US Patent 7.498.460, 2009) (Ozaki et al., 2015; Coll et al., 2011; Haerter et al., 2009) was described and found to inhibit both NLRP3 and AIM2 inflammasome-induced IL-1 $\beta$ processing, by preventing ASC oligomerization and it also appears to have further roles in innate immune responses, different from its role of adaptor for inflammasome formation (Ozaki et al., 2015). Therefore, this small-molecule inhibitor of ASC may hold therapeutic promise as a dual-purpose therapy in some inflammatory conditions (Coll et al., 2011). It has been reported the use of an anti-human ASC monoclonal antibody (clone 23-4, MBL, Nagoya, Japan) for ASC identification, cloning and characterization (Masumoto et al., 2001). Kaneko et al. (2015) have studied whether this anti-ASC human antibody would interfere with PYD of ASC and if it could then be considered a therapeutic candidate against disorders due to AIM2-inflammasome dysregulation. Since AIM2 is an intracellular receptor, enforced internalization of both ligands and candidate molecules is necessary for pharmacological screening. Interestingly, the authors developed a reconstituted in vitro AIM2 inflammasome in a cell-free system, which may serve as a useful tool to screen AIM2 inflammasometargeting therapeutics (Kaneko et al., 2015).

Inhibiting AIM2 inflammasome activity using synthetic inhibitors, such as synthetic Oligodeoxynucleotides (ODN) containing suppressive TTAGGG Motifs (Kaminski et al., 2013) or taking advantage of endogenous AIM2 inhibitors, such as the pyrin-containing proteins, recently described by (Khare et al., 2014; de Almeida et al., 2015), or antimicrobial cathelicidin peptides, reported by Schauber and colleagues (Dombrowski et al., 2011), could certainly be explored and studied for their potential to limit undesired inflammation (Man et al., 2016). Still, further research aiming at the discovery of new small-molecules to inhibit AIM2 inflammasome would certainly be of benefit to the development of effective drugs to treat chronic diseases, in which inflammation is the key player.

\section{Conclusion and Perspective}

Understanding the inflammasome activity is crucial for host response to microbes and possibly for ideal response to vaccine adjuvants (Guo et al., 2015). Given that, not all inflammasome activation can be considered harmful, it is very important to have a greater knowledge of the balance between beneficial and deleterious effects caused by inflammasome activation. For example, in the context of cancer, inflammasomes play pleiotropic roles since they could positively influence anti-cancer immuno-surveillance, but they can also induce carcinogenic inflammation, tumor growth, metastasis and angiogenesis. Due to such ambiguity, it is not possible to express definite advices to either inhibit or stimulate the inflammasomes or their products in cancer therapy: depending on the type of neoplasm, inflammasome inhibition maybe recommended as a therapeutic option or counter indicated (Guo et al., 2015). With regard to neurodegenerative diseases, such as Alzheimer's disease and multiple sclerosis, recently, it has been shown that the inflammasome modulates neuroinflammatory cells and the initial stages of neuroinflammation. The activation of the inflammasome, upon a subsequent cascade of events, correlated to neuro-inflammation (such as oxidative stress), makes it, indeed, a promising therapeutic target for the modulation of these disorders (Freeman and Ting, 2016).

However, over-activation of the sensing mechanisms for RNA and DNA recognition or the incapability to properly control and restrain the responses is directly linked to auto-immune disorders (Smith and Jefferies, 2014). As widely discussed herein, focusing on AIM2 inflammasome, it is known that aberrant recognition of cytoplasmic self-DNA by AIM2 leads to the development of several pathologies, like psoriasis, dermatitis, arthritis and other autoimmune and chronic inflammatory diseases. Therefore, here the need and the challenge of understanding and dissecting deeper the molecular mechanisms through which innate immune cells sense self-DNA and initiate an immuneresponse. This will enable to identify new therapeutic agents to effectively target AIM2 activated by dsDNA and consequently treat the related autoimmune/inflammatory diseases and conditions. In addition, further characterization of the neuronal AIM2 inflammasome and pyroptosis could also serve as an effective tool for the progression and the development of new therapies to control and restrain both the inappropriate autoimmune reactions to host nucleic acids and the pathologic sequelae of neuroinflammation.

\section{Acknowledgement}

I would like to thank Professor Guy Maddern for proofreading the manuscript. 


\section{Disclosure Statement}

The author declare no conflict of interest

\section{References}

Adamczak, S.E., J.P. de Rivero Vaccari, G. Dale, F.J. Brand, 3RD and D. Nonner et al., 2014. Pyroptotic neuronal cell death mediated by the AIM2 inflammasome. J. Cereb. Blood Flow Metab., 34: 621-9. DOI: 10.1038/jcbfm.2013.236

Apostolidis, S.A., L.A. Lieberman, K. Kis-Toth, J.C. Crispin and G.C. Tsokos, 2011. The dysregulation of cytokine networks in systemic lupus erythematosus. J. Interferon Cytokine Res., 31: 769-79. DOI: 10.1089/jir.2011.0029

Baccala, R., R. Gonzalez-Quintial, B.R. Lawson, M.E. Stern and D.H. Kono et al., 2009. Sensors of the innate immune system: Their mode of action. Nat. Rev. Rheumatol., 5: 448-56.

DOI: $10.1038 /$ nrrheum.2009.136

Baccala, R., K. Hoebe, D.H. Kono, B. Beutler and A.N. Theofilopoulos, 2007. TLR-dependent and TLR-independent pathways of type I interferon induction in systemic autoimmunity. Nat. Med., 13: 543-551. DOI: $10.1038 / \mathrm{nm} 1590$

Bakele, M., M. Joos, S. Burdi, N. Allgaier and S. Poschel et al., 2014. Localization and functionality of the inflammasome in neutrophils. J. Biol. Chem., 289: 5320-5329.

DOI: $10.1074 /$ jbc.M113.505636

Baldwin, A.G., D. Brough and S. Freeman, 2015. Inhibiting the Inflammasome: A Chemical Perspective. J. Med. Chem.

Baum, R., S. Sharma, S. Carpenter, Q.Z. Li and P. Busto et al., 2015. Cutting edge: AIM2 and endosomal TLRs differentially regulate arthritis and autoantibody production in DNase II-deficient mice. J. Immunol., 194: 873-877.

DOI: $10.4049 /$ jimmunol.1402573

Belhocine, K. and D.M. Monack, 2012. Francisella infection triggers activation of the AIM2 inflammasome in murine dendritic cells. Cell Microbiol., 14: 71-80.

DOI: $10.1111 / \mathrm{j} .1462-5822.2011 .01700 . \mathrm{x}$

Burckstummer, T., C. Baumann, S. Bluml, E. Dixit and G. Durnberger et al., 2009. An orthogonal proteomic-genomic screen identifies AIM2 as a cytoplasmic DNA sensor for the inflammasome. Nat. Immunol., 10: 266-272. DOI: 10.1038/ni.1702

Cai, X., J. Chen, H. Xu, S. Liu and Q.X. Jiang et al., 2014. Prion-like polymerization underlies signal transduction in antiviral immune defense and inflammasome activation. Cell, 156: 1207-1222.

DOI: $10.1016 /$ j.cell.2014.01.063
Campello Yurgel, V., N. Ikuta, A. Brondani da Rocha, V.R. Lunge and R. Fett Schneider et al., 2007. Role of plasma DNA as a predictive marker of fatal outcome following severe head injury in males. J. Neurotrauma, 24: 1172-1181. DOI: $10.1089 /$ neu.2006.0160

Chen, L.C., L.J. Wang, N.M. Tsang, D.M. Ojcius and C.C. Chen et al., 2012. Tumour inflammasomederived IL-1beta recruits neutrophils and improves local recurrence-free survival in EBV-induced nasopharyngeal carcinoma. EMBO Mol. Med., 4: 1276-1293. DOI: 10.1002/emmm.201201569

Choubey, D. and R. Panchanathan, 2008. Interferoninducible Ifi200-family genes in systemic lupus erythematosus. Immunol. Lett., 119: 32-41. DOI: $10.1016 /$ j.imlet.2008.06.001

Coll, R.C., A. Robertson, M. Butler, M. Cooper and L.A. O'neill, 2011. The cytokine release inhibitory drug CRID3 targets ASC oligomerisation in the NLRP3 and AIM2 inflammasomes. PLoS One, 6: e29539-e29539.

DOI: $10.1371 /$ journal.pone.0029539

Crane, D.D., T.J. Bauler, T.D. Wehrly and C.M. Bosio, 2014. Mitochondrial ROS potentiates indirect activation of the AIM2 inflammasome. Front Microbiol., 5: 438.

DOI: $10.3389 /$ fmicb. 2014.00438

Crispin, J.C., S.N. Liossis, K. Kis-Toth, L.A. Lieberman and V.C. Kyttaris et al., 2010. Pathogenesis of human systemic lupus erythematosus: Recent advances. Trends Mol. Med., 16: 47-57.

DOI: 10.1016/j.molmed.2009.12.005

de Almeida, L., S. Khare, A.V. Misharin, R. Patel and R.A. Ratsimandresy et al., 2015. The PYRIN domain-only protein POP1 inhibits inflammasome assembly and ameliorates inflammatory disease. Immunity, 43: 264-76.

DOI: 10.1016/j.immuni.2015.07.018

de Koning, H.D., J.G. Bergboer, E.H. van den Bogaard, I.M. van Vlijmen-Willems and D. Rodijk-Olthuis et al., 2012. Strong induction of AIM2 expression in human epidermis in acute and chronic inflammatory skin conditions. Exp. Dermatol., 21: 961-964. DOI: $10.1111 /$ exd.12037

de Rivero Vaccari, J.P., W.D. Dietrich and R.W. Keane, 2014. Activation and regulation of cellular inflammasomes: Gaps in our knowledge for central nervous system injury. J. Cereb. Blood Flow Metab., 34: 369-75. DOI: 10.1038/jcbfm.2013.227

Dihlmann, S., S. Tao, F. Echterdiek, E. Herpel and L. Jansen et al., 2014. Lack of Absent in Melanoma 2 (AIM2) expression in tumor cells is closely associated with poor survival in colorectal cancer patients. Int. J. Cancer, 135: 2387-2396. DOI: $10.1002 /$ ijc.28891 
Dombrowski, Y., S. Koglin and J. Schauber, 2012. DNAtriggered AIM2 inflammasome activation in keratinocytes: Comment on Kopfnagel et al. Exp Dermatol., 2011. 20: 1027-1029. Exp. Dermatol., 21: 474-475. DOI: 10.1111/j.1600-0625.2012.01466.x

Dombrowski, Y., M. Peric, S. Koglin, C. Kammerbauer and C. Goss et al., 2011. Cytosolic DNA triggers inflammasome activation in keratinocytes in psoriatic lesions. Sci. Translat. Med., 3: 82ra38-82ra38. DOI: $10.1126 /$ scitranslmed.3002001

Dotson, R.J., S.M. Rabadi, E.L. Westcott, S. Bradley and S.V. Catlett et al., 2013. Repression of inflammasome by Francisella tularensis during early stages of infection. J. Biol. Chem., 288: 23844-57. DOI: $10.1074 /$ jbc.M113.490086

Ekchariyawat, P., R. Hamel, E. Bernard, S. Wichit and P. Surasombatpattana et al., 2015. Inflammasome signaling pathways exert antiviral effect against Chikungunya virus in human dermal fibroblasts. Infect. Genet. Evol., 32: 401-408. DOI: 10.1016/j.meegid.2015.03.025

Fang, R., K. Tsuchiya, I. Kawamura, Y. Shen and H. Hara et al., 2011. Critical roles of ASC inflammasomes in caspase-1 activation and host innate resistance to Streptococcus pneumoniae infection. J. Immunol., 187: 4890-4899. DOI: $10.4049 /$ jimmunol.1100381

Feng, J., J. Park, P. Cron, D. Hess and B.A. Hemmings, 2004. Identification of a PKB/Akt hydrophobic motif Ser-473 kinase as DNA-dependent protein kinase. J. Biol. Chem., 279: 41189-41196. DOI: $10.1074 /$ jbc.M406731200

Fernandes-Alnemri, T., J.W. Yu, P. Datta, J. Wu and E.S. Alnemri, 2009. AIM2 activates the inflammasome and cell death in response to cytoplasmic DNA. Nature, 458: 509-513.

DOI: 10.1038 /nature07710

Fernandes-Alnemri, T., J.W. Yu, C. Juliana, L. Solorzano and S. Kang et al., 2010. The AIM2 inflammasome is critical for innate immunity to Francisella tularensis. Nat. Immunol., 11: 385-393. DOI: 10.1038/ni.1859

Fish, E.N., 2008. The X-files in immunity: Sex-based differences predispose immune responses. Nat. Rev. Immunol., 8: 737-744. DOI: 10.1038/nri2394

Flight, M.H., 2012. Deal watch: High hopes for oral SYK inhibitor in rheumatoid arthritis. Nat. Rev. Drug Discov., 11: 10-10. DOI: 10.1038/nrd3631

Freeman, L.C. and J.P. Ting, 2016. The pathogenic role of the inflammasome in neurodegenerative diseases. J. Neurochem., 136: 29-38.

Garcia-Romo, G.S., S. Caielli, B. Vega, J. Connolly and F. Allantaz et al., 2011. Netting neutrophils are major inducers of type I IFN production in pediatric systemic lupus erythematosus. Sci. Translat. Med., 3: 73ra20-73ra20.

DOI: $10.1126 /$ scitranslmed.3001201
Genovese, M.C., A. Kavanaugh, M.E. Weinblatt, C. Peterfy and J. Dicarlo et al., 2011. An oral Syk kinase inhibitor in the treatment of rheumatoid arthritis: A three-month randomized, placebo-controlled, phase II study in patients with active rheumatoid arthritis that did not respond to biologic agents. Arthritis Rheum., 63: 337-45. DOI: 10.1002/art.30114

Grimminger, F., R.T. Schermuly and H.A. Ghofrani, 2010. Targeting non-malignant disorders with tyrosine kinase inhibitors. Nat. Rev. Drug Discov., 9: 956-70. DOI: 10.1038/nrd3297

Guarda, G., M. Braun, F. Staehli, A. Tardivel and C. Mattmann et al., 2011. Type I interferon inhibits interleukin-1 production and inflammasome activation. Immunity, 34: 213-23.

DOI: $10.1016 /$ j.immuni.2011.02.006

Guo, H., J.B. Callaway and J.P. Ting, 2015. Inflammasomes: Mechanism of action, role in disease and therapeutics. Nat. Med., 21: 677-87. DOI: $10.1038 / \mathrm{nm} .3893$

Haerter, M., J. Ergueden, F. Wunder, H. Tinel and J. Koebberling et al. Bayer Healthcare, AG, A 2009, March 3. Isophthalic acid derivatives.

Hanamsagar, R., A. Aldrich and T. Kielian, 2014. Critical role for the AIM2 inflammasome during acute CNS bacterial infection. J. Neurochem., 129: 704-11. DOI: 10.1111/jnc. 12669

Hara, H., K. Tsuchiya, I. Kawamura, R. Fang and E. Hernandez-Cuellar et al., 2013. Phosphorylation of the adaptor ASC acts as a molecular switch that controls the formation of speck-like aggregates and inflammasome activity. Nat. Immunol., 14: 1247-1255. DOI: 10.1038/ni.2749

He, Y., S. Varadarajan, R. Munoz-Planillo, A. Burberry and Y. Nakamura et al., 2014. 3,4-methylenedioxybeta-nitrostyrene inhibits NLRP3 inflammasome activation by blocking assembly of the inflammasome. J. Biol. Chem., 289: 1142-1150. DOI: $10.1074 /$ jbc.M113.515080

Honda, H., Y. Nagai, T. Matsunaga, N. Okamoto and Y. Watanabe et al., 2014. Isoliquiritigenin is a potent inhibitor of NLRP3 inflammasome activation and dietinduced adipose tissue inflammation. J. Leukoc. Biol., 96: 1087-100. DOI: 10.1189/jlb.3A0114-005RR

Hornung, V., A. Ablasser, M. Charrel-Dennis, F. Bauernfeind and G. Horvath et al., 2009. AIM2 recognizes cytosolic dsDNA and forms a caspase-1activating inflammasome with ASC. Nature, 458: 514-518. DOI: 10.1038 /nature07725

Isakov, E., P. Weisman-Shomer and M. Benhar, 2014. Suppression of the pro-inflammatory NLRP3/interleukin-1beta pathway in macrophages by the thioredoxin reductase inhibitor auranofin. Biochim. Biophys. Acta, 1840: 3153-3161. DOI: 10.1016/j.bbagen.2014.07.012 
Jakobs, C., S. Perner and V. Hornung, 2015. AIM2 drives joint inflammation in a Self-DNA triggered model of chronic polyarthritis. PLoS One, 10: e0131702-e0131702. DOI:

10.1371/journal.pone.0131702

Javierre, B.M., A.F. Fernandez, J. Richter, F. Al-Shahrour and J.I. Martin-Subero et al., 2010. Changes in the pattern of DNA methylation associate with twin discordance in systemic lupus erythematosus. Genome Res., 20: 170-179.

DOI: $10.1101 /$ gr. 100289.109

Jin, T., A. Perry, P. Smith, J. Jiang and T.S. Xiao, 2013. Structure of the absent in melanoma 2 (AIM2) pyrin domain provides insights into the mechanisms of AIM2 autoinhibition and inflammasome assembly. J. Biol. Chem., 288: 13225-13235. DOI: $10.1074 /$ jbc.M113.468033

Juliana, C., T. Fernandes-Alnemri, J. Wu, P. Datta and L. Solorzano et al., 2010. Anti-inflammatory compounds parthenolide and Bay 11-7082 are direct inhibitors of the inflammasome. J. Biol. Chem., 285: 9792-802. DOI: 10.1074/jbc.M109.082305

Kahlenberg, J.M., S.G. Thacker, C.C. Berthier, C.D. Cohen and M. Kretzler et al., 2011. Inflammasome activation of IL-18 results in endothelial progenitor cell dysfunction in systemic lupus erythematosus. J. Immunol., 187: 6143-56. DOI: $10.4049 /$ jimmunol.1101284

Kalantari, P., R.B. Deoliveira, J. Chan, Y. Corbett and V. Rathinam et al., 2014. Dual engagement of the NLRP3 and AIM2 inflammasomes by plasmodiumderived hemozoin and DNA during malaria. Cell Rep., 6: 196-210.

DOI: $10.1016 /$ j.celrep.2013.12.014

Kaminski, J.J., S.A. Schattgen, T.C. Tzeng, C. Bode and D.M. Klinman et al., 2013. Synthetic oligodeoxynucleotides containing suppressive TTAGGG motifs inhibit AIM2 inflammasome activation. J. Immunol., 191: 3876-83.

DOI: 10.4049/jimmunol.1300530

Kaneko, N., Y. Ito, T. Iwasaki, H. Takeda and T. Sawasaki et al., 2015. Reconstituted AIM2 inflammasome in cell-free system. J. Immunol. Meth., 426: 76-81. DOI: 10.1016/j.jim.2015.08.004

Kanneganti, T.D., 2010. Central roles of NLRs and inflammasomes in viral infection. Nat. Rev. Immunol., 10: 688-98. DOI: 10.1038/nri2851

Kaplan, M.J., 2011. Neutrophils in the pathogenesis and manifestations of SLE. Nat. Rev. Rheumatol., 7: 691-9. DOI: 10.1038/nrrheum.2011.132

Karki, R., S.M. Man, R.K. Malireddi, P. Gurung and P. Vogel et al., 2015. Concerted activation of the AIM2 and NLRP3 inflammasomes orchestrates host protection against Aspergillus infection. Cell Host Microbe, 17: 357-68.

DOI: 10.1016/j.chom.2015.01.006
Kawane, K., H. Fukuyama, G. Kondoh, J. Takeda and Y. Ohsawa et al., 2001. Requirement of DNase II for definitive erythropoiesis in the mouse fetal liver. Science, 292: 1546-1549. DOI: $10.1126 /$ science.292.5521.1546

Khare, S., R.A. Ratsimandresy, L. de Almeida, C.M. Cuda and S.L. Rellick et al., 2014. The PYRIN domain-only protein POP3 inhibits ALR inflammasomes and regulates responses to infection with DNA viruses. Nat. Immunol., 15: 343-53. DOI: $10.1038 /$ ni.2829

Kim, S., F. Bauernfeind, A. Ablasser, G. Hartmann and K.A. Fitzgerald et al., 2010. Listeria monocytogenes is sensed by the NLRP3 and AIM2 inflammasome. Eur. J. Immunol., 40: 1545-51. DOI: $10.1002 /$ eji.201040425

Kimkong, I., Y. Avihingsanon and N. Hirankarn, 2009. Expression profile of HIN200 in leukocytes and renal biopsy of SLE patients by real-time RT-PCR. Lupus, 18: 1066-72. DOI: $10.1177 / 0961203309106699$

Kondo, Y., K. Nagai, S. Nakahata, Y. Saito and T. Ichikawa et al., 2012. Overexpression of the DNA sensor proteins, absent in melanoma 2 and interferon-inducible 16 , contributes to tumorigenesis of oral squamous cell carcinoma with p53 inactivation. Cancer Sci., 103: 782-90. DOI: $10.1111 / \mathrm{j} .1349-7006.2012 .02211 . \mathrm{x}$

Kong, H., Y. Wang, X. Zeng, Z. Wang and H. Wang et al., 2015. Differential expression of inflammasomes in lung cancer cell lines and tissues. Tumour Biol., 36: 7501-13. DOI: $10.1007 / \mathrm{s} 13277-015-3473-4$

Kopfnagel, V., M. Wittmann and T. Werfel, 2011. Human keratinocytes express AIM2 and respond to dsDNA with IL-1beta secretion. Exp. Dermatol., 20: 1027-9. DOI: 10.1111/j.1600-0625.2011.01382.x

Lamkanfi, M. and V.M. Dixit, 2012. Inflammasomes and their roles in health and disease. Ann. Rev. Cell Dev. Biol., 28: 137-61. DOI: 10.1146/annurevcellbio-101011-155745

Lande, R., D. Ganguly, V. Facchinetti, L. Frasca and C. Conrad et al., 2011. Neutrophils activate plasmacytoid dendritic cells by releasing self-DNApeptide complexes in systemic lupus erythematosus. Sci. Translat. Med., 3: 73ra19-73ra19. DOI: $10.1126 /$ scitranslmed.3001180

Laudisi, F., E. Vigano and A. Mortellaro, 2014. Tyrosine kinases: The molecular switch for inflammasome activation. Cell Mol. Immunol., 11: 129-31. DOI: $10.1038 / \mathrm{cmi} .2014 .4$

Liu, R., A.D. Truax, L. Chen, P. Hu and Z. Li et al., 2015. Expression profile of innate immune receptors, NLRs and AIM2, in human colorectal cancer: Correlation with cancer stages and inflammasome components. Oncotarget, 6: 33456-69. 
Lu, A., V.G. Magupalli, J. Ruan, Q. Yin and M.K. Atianand et al., 2014. Unified polymerization mechanism for the assembly of ASC-dependent inflammasomes. Cell, 156: 1193-206.

DOI: $10.1016 /$ j.cell.2014.02.008

Lu, D., J. Huang and A. Basu, 2006. Protein kinase C $\square$ activates protein kinase $\mathrm{B} / \mathrm{Akt}$ via DNA-PK to protect against tumor necrosis factor- $\alpha$-induced cell death. J. Biol. Chem., 281: 22799-807. DOI: $10.1074 /$ jbc.M603390200

Lupfer, C., A. Malik and T.D. Kanneganti, 2015. Inflammasome control of viral infection. Curr. Opin. Virol., 12: 38-46. DOI: 10.1016/j.coviro.2015.02.007

Maier, N.K., S.H. Leppla and M. Moayeri, 2015. The cyclopentenone prostaglandin $15 \mathrm{~d}-\mathrm{PGJ} 2$ inhibits the NLRP1 and NLRP3 inflammasomes. J. Immunol., 194: 2776-85. DOI: 10.4049/jimmunol.1401611

Man, S.M., L.J. Hopkins, E. Nugent, S. Cox and I.M. Gluck et al., 2014. Inflammasome activation causes dual recruitment of NLRC4 and NLRP3 to the same macromolecular complex. Proc. Nat. Acad. Sci. USA., 111: 7403-8. DOI: 10.1073/pnas.1402911111

Man, S.M. and T.D. Kanneganti, 2015. Regulation of inflammasome activation. Immunol. Rev., 265: 6-21. DOI: 10.1111/imr.12296

Man, S.M., R. KARKI and T.D. Kanneganti, 2016. AIM2 inflammasome in infection, cancer and autoimmunity: Role in DNA sensing, inflammation and innate immunity. Eur. J. Immunol., 46: 269-80. DOI: 10.1002/eji.201545839

Man, S.M., R. Karki, R.K. Malireddi, G. Neale and P. Vogel et al., 2015a. The transcription factor IRF1 and guanylate-binding proteins target activation of the AIM2 inflammasome by Francisella infection. Nat. Immunol., 16: 467-75. DOI: 10.1038/ni.3118

Man, S.M., Q. Zhu, L. Zhu, Z. Liu and R. Karki et al., 2015b. Critical role for the DNA sensor AIM2 in stem cell proliferation and cancer. Cell, 162: 45-58. DOI: $10.1016 /$ j.cell.2015.06.001

Marshak-Rothstein, A., 2006. Toll-like receptors in systemic autoimmune disease. Nat. Rev. Immunol., 6: 823-35. DOI: 10.1038/nri1957

Masumoto, J., S. Taniguchi and J. Sagara, 2001. Pyrin N-terminal homology domain- and caspase recruitment domain-dependent oligomerization of ASC. Biochem. Biophys. Res. Commun., 280: 652-5. DOI: $10.1006 /$ bbrc. 2000.4190

Miao, E.A., J.V. Rajan and A. Aderem, 2011. Caspase1 -induced pyroptotic cell death. Immunol. Rev., 243: 206-14.

DOI:10.1111/j.1600-065X.2011.01044.X

Munoz, L.E., C. Janko, C. Schulze, C. Schorn and K. Sarter et al., 2010. Autoimmunity and chronic inflammation-two clearance-related steps in the etiopathogenesis of SLE. Autoimmun. Rev., 10: 38-42. DOI: 10.1016/j.autrev.2010.08.015
Ozaki, E., M. Campbell and S.L. Doyle, 2015. Targeting the NLRP3 inflammasome in chronic inflammatory diseases: Current perspectives. J. Inflamm. Res., 8: 15-27.

Ponomareva, L., H. Liu, X. Duan, E. Dickerson and H. Shen et al., 2013. AIM2, an IFN-inducible cytosolic DNA sensor, in the development of benign prostate hyperplasia and prostate cancer. Mol. Cancer Res., 11: 1193-202. DOI: 10.1158/1541-7786.MCR-13-0145

Rathinam, V.A., Z. Jiang, S.N. Waggoner, S. Sharma and L.E. Cole et al., 2010. The AIM2 inflammasome is essential for host defense against cytosolic bacteria and DNA viruses. Nat. Immunol., 11: 395-402. DOI: 10.1038/ni.1864

Reboldi, A., E.V. Dang, J.G. Mcdonald, G. Liang and D.W. Russell et al., 2014. 25-Hydroxycholesterol suppresses interleukin-1-driven inflammation downstream of type I interferon. Science, 345: 679-84. DOI: 10.1126/science. 1254790

Reinholz, M., Y. Kawakami, S. Salzer, A. Kreuter and Y. Dombrowski et al., 2013. HPV16 activates the AIM2 inflammasome in keratinocytes. Arch Dermatol. Res., 305: 723-32. DOI: $10.1007 / \mathrm{s} 00403-013-1375-0$

Roberts, T.L., A. Idris, J.A. Dunn, G.M. Kelly and C.M. Burnton et al., 2009. HIN-200 proteins regulate caspase activation in response to foreign cytoplasmic DNA. Science, 323: 1057-60. DOI: $10.1126 /$ science. 1169841

Rudolphi, K., N. Gerwin, N. Verzijl, P. van der Kraan and W. van den Berg, 2003. Pralnacasan, an inhibitor of interleukin-1beta converting enzyme, reduces joint damage in two murine models of osteoarthritis. Osteoarthritis Cartilage, 11: 738-46. DOI: $10.1016 / \mathrm{S} 1063-4584(03) 00153-5$

Schroder, K. and J. Tschopp, 2010. The inflammasomes. Cell, 140: 821-32. DOI: 10.1016/j.cell.2010.01.040

Smith, S. and C. Jefferies, 2014. Role of DNA/RNA sensors and contribution to autoimmunity. Cytokine Growth Factor Rev., 25: 745-57. DOI: 10.1016/j.cytogfr.2014.07.019

Strowig, T., J. Henao-Mejia, E. Elinav and R. Flavell, 2012. Inflammasomes in health and disease. Nature, 481: 278-86. DOI: 10.1038/nature 10759

Ting, J.P., R.C. Lovering, E.S. Alnemri, J. Bertin and J.M. Boss et al., 2008. The NLR gene family: A standard nomenclature. Immunity, 28: 285-7. DOI: 10.1016/j.immuni.2008.02.005

Tsuchiya, K., H. Hara, I. Kawamura, T. Nomura and T. Yamamoto et al., 2010. Involvement of absent in melanoma 2 in inflammasome activation in macrophages infected with Listeria monocytogenes. J. Immunol., 185: 1186-95. DOI: $10.4049 /$ jimmunol.1001058 
van der Pouw Kraan, T.C., C.A. Wijbrandts, L.G. van Baarsen, A.E. Voskuyl and F. Rustenburg et al., 2007. Rheumatoid arthritis subtypes identified by genomic profiling of peripheral blood cells: Assignment of a type I interferon signature in a subpopulation of patients. Ann Rheum Dis., 66: 1008-1014. DOI: 10.1136/ard.2006.063412

Vanaja, S.K., V.A. Rathinam and K.A. Fitzgerald, 2015. Mechanisms of inflammasome activation: Recent advances and novel insights. Trends Cell Biol., 25: 308-15. DOI: $10.1016 /$ j.tcb.2014.12.009

Wang, L.J., C.W. Hsu, C.C. Chen, Y. Liang and L.C. Chen et al., 2012. Interactome-wide analysis identifies end-binding protein 1 as a crucial component for the speck-like particle formation of activated Absence in Melanoma 2 (AIM2) inflammasomes. Mol. Cell Proteom., 11: 1230-44. DOI: $10.1074 / \mathrm{mcp} . M 112.020594$

Warren, S.E., A. Armstrong, M.K. Hamilton, D.P. Mao and I.A. Leaf et al., 2010. Cutting edge: Cytosolic bacterial DNA activates the inflammasome via Aim2. J. Immunol., 185: 818-21. DOI: 10.4049/jimmunol.1000724

Wilson, J.E., A.S. Petrucelli, L. Chen, A.A. Koblansky and A.D. Truax et al., 2015. Inflammasomeindependent role of AIM2 in suppressing colon tumorigenesis via DNA-PK and Akt. Nat. Med., 21: 906-13. DOI: $10.1038 / \mathrm{nm} .3908$
Yang, C.A., S.T. Huang and B.L. Chiang, 2015. Sexdependent differential activation of NLRP3 and AIM2 inflammasomes in SLE macrophages. Rheumatology, 54: 324-31.

DOI: $10.1093 /$ rheumatology/keu3 18

Yoshida, H., Y. Okabe, K. Kawane, H. Fukuyama and S. Nagata, 2005. Lethal anemia caused by interferonbeta produced in mouse embryos carrying undigested DNA. Nat. Immunol., 6: 49-56. DOI: $10.1038 /$ ni1 146

Zhen, J., L. Zhang, J. Pan, S. Ma and X. Yu et al., 2014. AIM2 mediates inflammation-associated renal damage in hepatitis B virus-associated glomerulonephritis by regulating caspase-1, ILlbeta and IL-18. Mediators Inflamm., 2014: 190860-190868. DOI: 10.1155/2014/190860

Zitvogel, L., O. Kepp, L. Galluzzi and G. Kroemer, 2012. Inflammasomes in carcinogenesis and anticancer immune responses. Nat. Immunol., 13: 343-51. DOI: $10.1038 /$ ni.2224 